\title{
Immunotherapy for recurrent orthostatic cerebral hypo-perfusion syndrome in recurrent COVID-19 infection
}

\author{
Hugues Ghislain Atakla $M D^{1}$ iD, Mahugnon Maurel Ulrich Dénis Noudohounsi $M D^{2}$ iD, Fatoumata Lounceny \\ Barry $M D^{3}$ (iD), Carlos Othon Guelngar $M D^{4}$ iD , Fodé Abass Cissé $M D^{5}$ iD, Dismand Stephan Houinato $M D^{6}$ iD \\ ${ }^{1,6}$ Neurology Department, University Hospital Center, Hubert Koutougou MAGA, Cotonou, Benin \\ ${ }^{1,6}$ Laboratory of Non-communicable and Neurologic Diseases Epidemiology, Faculty of Health Science, University of \\ Abomey-Calavi, Cotonou, Benin \\ ${ }^{2}$ Monitoring and evaluation/WHO AFRO, Research Project Manager, Brazzaville, Congo \\ ${ }^{3}$ Neurosurgery Department, University Hospital Center, Conakry, Guinea \\ ${ }^{4,5}$ Neurology Department, Ignace Deen University Hospital Center, Conakry, Guinea
}

Date of submission: $9^{\text {th }}$ March 2021

Date of acceptance: $5^{\text {th }}$ May 2021

Date of publication: $1^{\text {st }}$ June 2021

\begin{abstract}
Orthostatic hypotension, often interpreted as a fall in blood pressure as a person moves from lying or sitting to standing, has a direct impact on the brain. It results in a decrease in brain perfusion pressure and in many cases causes symptoms of transient cerebral hypoperfusion. This report treats a case of recurrent orthostatic hypotension in a field of recurrent COVID-19 infection, with a good clinical response to early immunotherapy. Signs of lung infection retroceded until complete disappearance under treatment with chloroquine sulfate and azithromycin. However, due to persistent signs of dysautonomy and paresthesias, the patient was subjected to intravenous immunoglobulin immunotherapy with good therapeutic response until the patient's complete recovery 150 days after the start of treatment. The prognosis remains unknown at the time, as data is not yet available from large-scale studies on this subject.
\end{abstract}

Key words: Brain hypoperfusion, Immunotherapy, Recurrent, SARS-CoV-2.

\section{Introduction}

Oror oronavirus (COVID-19) is one of the main viruses that target the human respiratory system, but it also has neuro-invasive capabilities, ${ }^{1}$ and can spread from the respiratory tract to other body systems. COVID-19 disease presents primarily as acute respiratory syndrome with

Access this article online
Website: https://www.nepjol.info/index.php/NJN
DOI: https://doi.org/10.3126/njn.v18i2.35516
HOW TO CITE
Atakla HG, Noudohounsi MMUD, Barry FL, Gueingar CU,
Cissé FA, Houinato DS. Immunotherapy for recurrent orthostatic
cerebral hypo-perfusion syndrome in recurrent COVID-19
infection. NJNS. 2021;18(2):64-6.

Address for correspondence:

Hugues Ghislain Atakla

Neurology Department, University Hospital Center

Hubert Koutougou MAGA, Cotonou, Benin.

E-mail: neuroscience.ghislain@gmail.com

Phone: +22997492211

Copyright (C) 2021 Nepalese Society of Neurosurgeons (NESON)

ISSN: 1813-1948 (Print), 1813-1956 (Online)

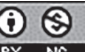

This work is licensed under a Creative Commons Attribution-Non Commercial 4.0 International License. fever, fatigue, dry cough, myalgia and dyspnea. There is also evidence of involvement of other structures including neurological manifestations that have been reported by several authors. ${ }^{2,3,4}$ However, among these neurological manifestations, disorders of the autonomic nervous system (ANS) with overactivity of the parasympathetic nervous system (PNS) on the sympathetic nervous system (SNS) have been very little reported. This disorder is commonly manifested by: dizziness; chronic fatigue, orthostatic vertigo, cerebral fog and neck pain. Several etiologies are known but the list of etiologies seems to be growing with the advent of COVID-19 caused by SARS-CoV-2. The latter targets the respiratory system but also a large part of the body's systems including the central nervous system (CNS) and peripheral nervous system (PNS). The involvement of an autoimmune mechanism in SARS$\mathrm{CoV}-2$ infection has been suggested in this observation. We describe in this report a case of recurrent orthostatic hypotension in a field of recurrent COVID-19 infection, with a good clinical response to early immunotherapy.

\section{Case Report}

A 52-year-old woman with apyretic symptoms presented for a neurological consultation for leg pain, tingling paresthesia and burns in the lower and upper 
limbs that had been present for 72 hours on admission. The anamnesis reveals a personal history of COVID-19 infection treated and cured about a month ago. There had been no recent known exposure to COVID-19. The general and neurological examination of the patient revealed no notable particularities. Similarly, the complete biological assessment with ionogram and electro-neuro-myogram (ENMG) did not provide any explanation for the patient's symptoms. She received a prescription based on a tricyclic antidepressant (Laroxyl gout 40mg) with a vitamin complex (Neurobion) and an appointment in 14 days. During the 72 hours of the first consultation, the patient came back for dizziness, chronic fatigue, ringing in the ears, cervical pain and pain in the cephalic end, all of them with an orthostatic character in a febrile context $\left(38^{\circ} \mathrm{C}\right)$. Blood pressure (BP) measurement in supine position was normal while sitting and standing position showed an abrupt collapse of systolic (SBP) and diastolic blood pressure (DBP): 90/60 $\mathrm{mm}$ of $\mathrm{Hg}$ and $70 / 40 \mathrm{~mm}$ of $\mathrm{Hg}$ respectively. No pallor or compensatory tachycardia were noted. Cerebral blood flow speed could not be appreciated because of unavailability of transcranial Doppler. On the basis of clinical elements, a diagnosis of orthostatic cerebral hypoperfusion with sensory polyneuropathies was suggested and the patient was put under observation for an etiological investigation. The cardiac assessment was normal and the search for other pathologies, particularly neurological and familial, with a degenerative appearance was not productive.

In addition, worsening of the patient's condition was noticed with onset of cough, dyspnea and hypoxemia. The patient was transferred to the intensive care unit where she was given high-flow oxygen and non-invasive ventilation pending a positive SARS-CoV-2 reverse transcriptase PCR (RT-PCR) result. At this stage, the repeated bioassay revealed a biological inflammatory syndrome with a CRP of $38 \mathrm{mg} / \mathrm{dl}$ and lymphopenia. The D-dimer was $11644 \mu \mathrm{g} / \mathrm{L}$. Thoracic CT scan showed viral pneumonia (Figure 1).

Appropriate sanitary measures were taken and the patient was subjected to chloroquine sulfate in combination with azithromycin. After 10 days of treatment, the clinical respiratory signs totally regressed with more or less stationary persistence of dysautonomic signs and paresthesias. Following two repeated tests for SARS$\mathrm{CoV}-2$, which were negative; and the unsuccessful search for other causes of dysautonomic disorders, we suspected that an autoimmune mechanism was at the origin of the symptoms. The patient was treated with intravenous immunoglobulin at a dose of $2 \mathrm{~g} / \mathrm{kg} /$ month for two months which was reduced to a dose of $1 \mathrm{~g} / \mathrm{kg} /$ week. It should be noted that orthostatic vertigo, headache, paresthesia and leg pain regressed within 60 days after initiation of immunotherapy. 150 days after the onset of the symptoms of dysautonomy, the patient has no sequelae of the disease.

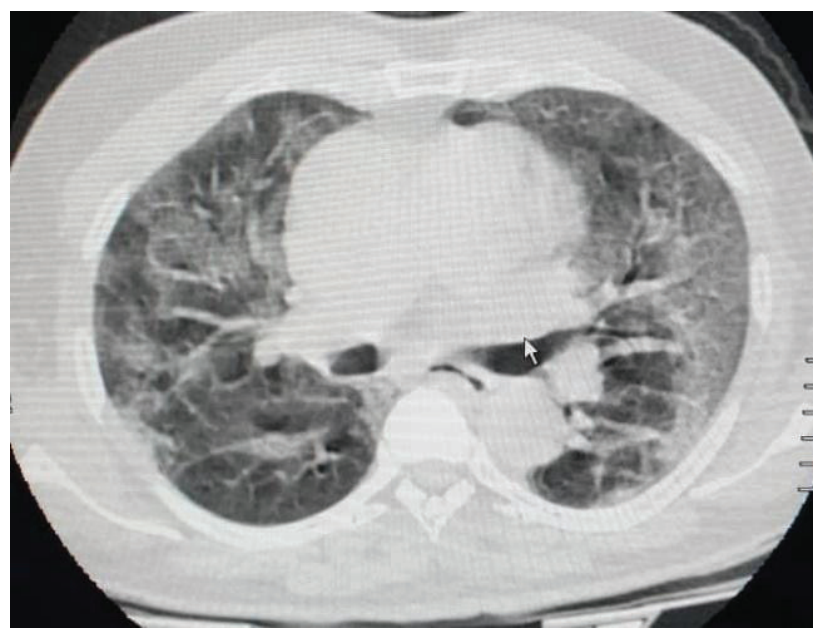

Figure 1: Bilateral diffuse frosted glass infiltrates

\section{Discussion}

This report describes a case of orthostatic hypotension syndrome in a patient with recurrent COVID-19 infection with a good response to immunotherapy. This case is the first observation in our country since the first case of SARS-CoV-2 infection recorded in our country. The onset of orthostatic hypotension was preceded by sensory disturbances such as paresthesia of the limbs. This peripheral nervous system dysfunction with dysautonomic disorders in the context of COVID-19 infection was also recently reported by Peter Novak. ${ }^{5}$ This disorder suggests an early damage of the peripheral nerve roots. In addition, we also noticed clinical signs of cerebral hypoperfusion, which led us to conclude that the CNS was simultaneously affected, even though we were not able to perform a transcranial Doppler scan in order to assess the speed of the blood flow. Nevertheless, there are several recent studies that support the thesis that the nervous system in general is affected by SARS-CoV-2 aggression. ${ }^{6,7}$

Whatever the cause, it is always a failure of the physiological mechanisms of blood pressure regulation involved in the transition to orthostatism. ${ }^{8}$ According to currently available data, orthostatic hypotension has rarely been associated with an infectious pathology of viral origin. However, several authors report cases of peripheral neuropathy or dysautonomia in the setting of SARS-CoV-2 infection or post COVID-19 infection. ${ }^{2,3,5,8}$ As a rule, orthostatic hypotension is related to drug intake, prolonged decubitus in the elderly subject, bulky varicose veins, hypovolemia, ${ }^{9-11}$ of which the etiological research in our case revealed none of these. Considering the history of our patient, other cases of dysautonomia of infectious origin reported in the literature, and the biological evidence that testifies the cytokine cascade following the viral aggression, we believe that an autoimmune reaction may have been triggered, secondary to the recurrence of the SARS-CoV-2 infection. We have therefore established 


\section{Atakla et al}

that the autoimmune reaction may have been the cause of the dysautonomia, thus affecting the vessels and/or baroreceptors in their roles of routing/serving the brain and systemic structures. Some recently described cases, such as peripheral neuropathies including Guillain-Barré polyradiculoneuritis (GBS) ${ }^{12}$ induced by SARS-CoV-2 infection, lead to the same conclusion. Moreover, the rapid therapeutic response to immunotherapy suggests the same mechanism as in GBS. Immunoglobulin at the dose described above resulted in complete resolution of symptoms by the 4th month of treatment. However, the subject deserves further reflection, by a large-scale investigation to better define it and establish a fixed therapeutic consensus.

\section{Conclusion}

In summary, this report describes an orthostatic cerebral hypo-perfusion and sensory neuropathy following an SARS-CoV-2 infection, adding to the large list of postinfection complications of COVID-19. The autoimmune reaction seems to be the mechanism involved and the conduct of good immunotherapy could facilitate the total regression of symptoms.

\section{Conflict of Interest: None}

Source(s) of support: None

\section{References}

1. Asadi-Pooya AA, Simani L. Central nervous system manifestations of COVID-19: A systematic review. Journal of the Neurological Sciences. 2020;413:16832. https://doi.org/10.1016/j.jns.2020.116832

2. Montalvan V, Lee J, Bueso T, et al. Neurological manifestations of COVID-19 and other coronavirus infections: A systematic review. Clin Neurol Neurosurg. 2020;194:105921.https://doi. org/10.1016/j.clineuro.2020.105921

3. Eshak N, Abdelnabi M, Ball S. Dysautonomia: An overlooked neurological manifestation in a critically ill COVID-19 patient. Am J Med Sci. 2020;360(4):4279.https://doi.org/10.1016/j.amjms.2020.07.022
4. Atakla HG, Condé $\mathrm{K}$, Neishay $\mathrm{A}$, et al. Cerebrovascular accidents indicative of COVID-19 infection: About 4 observations in Guinea: Accidents vasculaires cérébraux révélateurs d'infection au COVID-19: A propos de 4 observations en Guinée. Pan Africa Medical Journal. 2020, 35(2):65.https:// doi.org/10.11604/pamj.supp.2020.35.2.23751

5. Novak P. Post COVID-19 syndrome associated with orthostatic cerebral hypoperfusion syndrome, small fiber neuropathy and benefit of immunotherapy: A case report. eNeurological Sci. 2020;21:100276. https://doi.org/10.1016/j.ensci.2020.100276

6. Montalvan V, Lee J, Bueso T, et al. Neurological manifestations of COVID-19 and other coronavirus infections: A systematic review. Clin Neurol Neurosurg. 2020;194:105921.

7. Kaufmann H, Norcliffe-Kaufmann L, Palma JA. Baroreflex dysfunction. N Engl J Med. 2020;382(2):163-78.https://doi.org/10.1056/ NEJMra1509723

8. Hugues Ghislain Atakla, et al. Acute GuillainBarré polyradiculoneuritis indicative of COVID-19 infection: A case report. Pan African Medical Journal. 2020;35(2):150.https://doi.org/10.11604/pamj. supp.2020.35.150.25745

9. Danziger N, Alamowitch S. Neurologie 12e édition actualisée, 12th ed. Paris: Med-Line Editions;2018. ISBN 978-2-84678-232-6

10. Güler $\mathrm{S}$, et al. Intracranial hypotension is a rare cause of orthostatic headache: A review of the etiology, treatment and prognosis of 13 cases. Journal of the Neurological Sciences. 2013;333:e481-e518.https:// doi.org/10.1016/j.jns.2013.07.1706

11. Raffoul J, et al. A rare cause of recurrent orthostatic hypotension refractory to conventional treatment. American college of cardiology. JACC. 2016;67(13). https://doi.org/10.1016/S0735-1097(16)31251-7

12. Dalakas MC. Guillain-Barré syndrome: the first documented COVID-19-triggered autoimmune neurologic disease: more to come with myositis in the offing, Neurology, Neuroimmunology \& Neuroinflammation. American Academy of Neurology. 2020;5: e781.https://doi.org/10.1212/ NXI.0000000000000781 